\title{
SOME REFINEMENTS OF CLASSICAL INEQUALITIES
}

\author{
SHIGERU FURUICHI ${ }^{1}$ AND HAMID REZA MORADI ${ }^{2}$
}

\begin{abstract}
We give some new refinements and reverses Young inequalities in both additivetype and multiplicative-type for two positive numbers/operators. We show our advantages by comparing with known results. A few applications are also given. Some results relevant to the Heron mean are also considered.
\end{abstract}

\section{Introduction and Preliminaries}

In this paper, an operator means a bound linear operator on a Hilbert space $\mathcal{H}$. An operator $X$ is said to be positive (denoted by $X \geq 0$ ) if $\langle X y, y\rangle \geq 0$ for all $y \in \mathcal{H}$, and also an operator $X$ is said to be strictly positive (denoted by $X>0$ ) if $X$ is positive and invertible. For convenience, we often use the following notations:

$$
\begin{aligned}
& A !_{v} B \equiv\left((1-v) A^{-1}+v B^{-1}\right)^{-1}, \quad A \sharp_{v} B \equiv A^{\frac{1}{2}}\left(A^{-\frac{1}{2}} B A^{-\frac{1}{2}}\right)^{v} A^{\frac{1}{2}}, \\
& H_{v}(A, B) \equiv \frac{A \sharp_{v} B+A \sharp_{1-v} B}{2}, \quad A \nabla_{v} B \equiv(1-v) A+v B,
\end{aligned}
$$

where $A, B$ are strictly positive operators and $0 \leq v \leq 1$. When $v=\frac{1}{2}$, we write $A ! B, A \sharp B$, $H(A, B)$ and $A \nabla B$ for brevity, respectively.

A fundamental inequality between positive real numbers $a, b$ is the Young inequality, which states

$$
a^{1-v} b^{v} \leq(1-v) a+v b \quad 0 \leq v \leq 1,
$$

with equality if and only if $a=b$. If $v=\frac{1}{2}$, we obtain the arithmetic-geometric mean inequality $\sqrt{a b} \leq \frac{a+b}{2}$. Recently, a considerable attention is dedicated to the study of Young inequalities and its operator versions $[20,21]$.

It is well-known that, cf. [12]:

$$
A !_{v} B \leq A \sharp_{v} B \leq A \nabla_{v} B \quad 0 \leq v \leq 1,
$$

where the second inequality in (1.1) is known as the operator arithmetic-geometric mean inequality (or the operator Young inequality).

2010 Mathematics Subject Classification. Primary 47A63, Secondary 46L05, 47A60.

Key words and phrases. Operator inequality; Hermite-Hadamard inequality; Young inequality; Heron mean. 
Based on the refined scalar Young inequality, Kittaneh and Manasrah [14] obtained that

$$
r(A+B-2 A \sharp B)+A \sharp_{v} B \leq A \nabla_{v} B \leq R(A+B-2 A \sharp B)+A \sharp_{v} B,
$$

where $r=\min \{v, 1-v\}$ and $R=\max \{v, 1-v\}$.

Zou et al. [24] refined operator Young inequality with the Kantorovich constant $K(x) \equiv$ $\frac{(x+1)^{2}}{4 x},(x>0)$, and proposed the following result:

$$
K^{r}(h) A \sharp_{v} B \leq A \nabla_{v} B,
$$

where $0<\alpha^{\prime} I \leq A \leq \alpha I \leq \beta I \leq B \leq \beta^{\prime} I$ or $0<\alpha^{\prime} I \leq B \leq \alpha I \leq \beta I \leq A \leq \beta^{\prime} I, h=\frac{\beta}{\alpha}$ and $h^{\prime}=\frac{\beta^{\prime}}{\alpha^{\prime}}$. Note also that the inequality (1.3) improves Furuichi's result from [11], which includes the well known Specht's ratio instead of Kantorovich constant.

As for the reverse of the operator Young inequality, under the same conditions, Liao et al. [15] gave the following inequality:

$$
A \nabla_{v} B \leq K^{R}(h) A \sharp_{v} B .
$$

For more related inequalities and applications, see, e.g., [8, 9, 20, 21].

This paper intends to give some refinements and reverses for the operator Young inequality via Hermite-Hadamard inequality. That is, the following theorem is one of the main results in this paper.

Theorem A. Let $A, B$ be strictly positive operators such that $0<h^{\prime} I \leq A^{-\frac{1}{2}} B A^{-\frac{1}{2}} \leq h I \leq I$ for some positive scalars $h$ and $h^{\prime}$. Then for each $0 \leq v \leq 1$,

$$
m_{v}(h) A \sharp_{v} B \leq A \nabla_{v} B \leq M_{v}\left(h^{\prime}\right) A \sharp_{v} B,
$$

where

$$
m_{v}(x) \equiv 1+\frac{2^{v} v(1-v)(x-1)^{2}}{(x+1)^{v+1}},
$$

and

$$
M_{v}(x) \equiv 1+\frac{v(1-v)(x-1)^{2}}{2 x^{v+1}} .
$$

The proof of Theorem A is given in Section 2, and its advantage for previously known results are also given by Proposition 3.1 in Section 3.

To state our second main result, we recall that the family of Heron mean [1] for two positive numbers $a$ and $b$ is defined as

$$
F_{r, v}(a, b) \equiv r a^{1-v} b^{v}+(1-r)\{(1-v) a+v b\}, \quad 0 \leq v \leq 1 \text { and } r \in \mathbb{R} .
$$

More recently the first author [10] showed that if $r \leq 1$, then

$$
\left((1-v) a^{-1}+v b^{-1}\right)^{-1} \leq F_{r, v}(a, b), \quad 0 \leq v \leq 1 .
$$


Theorem B. Let $a, b \geq 0, r \in \mathbb{R}$ and $0 \leq v \leq 1$. Define

$$
\begin{gathered}
g_{r, v}(a, b) \equiv v\left(\frac{b-a}{a}\right)\left\{r\left(\frac{a+b}{2 a}\right)^{v-1}+(1-r)\right\}+1, \\
G_{r, v}(a, b) \equiv \frac{v}{2}\left(\frac{b-a}{a}\right)\left\{r a^{1-v} b^{v-1}+2-r\right\}+1 .
\end{gathered}
$$

(1) If either $a \leq b, r \geq 0$ or $b \leq a, r \leq 0$, then

$$
g_{r, v}(a, b) \leq F_{r, v}(a, b) \leq G_{r, v}(a, b) .
$$

(2) If either $a \leq b, r \leq 0$ or $b \leq a, r \geq 0$, then

$$
G_{r, v}(a, b) \leq F_{r, v}(a, b) \leq g_{r, v}(a, b) .
$$

We show the proof of Theorem B along with its advantage by four propositions in Section 4.

\section{On Refined Young Inequalities and Reverse Inequalities}

To achieve our results, we need the well-known Hermite-Hadamard inequality which asserts that if $f:[a, b] \rightarrow \mathbb{R}$ is a convex (concave) function, then the following chain of inequalities hold:

$$
f\left(\frac{a+b}{2}\right) \leq(\geq) \frac{1}{b-a} \int_{a}^{b} f(x) d x \leq(\geq) \frac{f(a)+f(b)}{2} .
$$

Our first attempt, which is a direct consequence of [18, Theorem 1], gives an additive-type improvement and reverse for the operator Young inequality via (2.1).

To obtain inequalities for bounded self-adjoint operators on Hilbert space, we shall use the following monotonicity property for operator functions: If $X \in \mathcal{B}(\mathcal{H})$ is a self-adjoint operator with a spectrum $S p(X)$ and $f, g$ are continuous real-valued functions on $S p(X)$, then

$$
f(t) \leq g(t), t \in S p(X) \quad \Rightarrow \quad f(X) \leq g(X) .
$$

The next lemma provides a technical result which we will need in the sequel.

Lemma 2.1. Let $0<v \leq 1$.

(i) For each $t>0$, the function $f_{v}(t)=v\left(1-t^{v-1}\right)$ is concave.

(ii) The function $g_{v}(t)=\frac{v(1-v)(t-1)}{t^{v+1}}$, is concave if $t \leq 1+\frac{2}{v}$, and convex if $t \geq 1+\frac{2}{v}$.

Proof. The function $f_{v}(t)$ is twice differentiable and $f_{v}^{\prime \prime}(t)=v(1-v)(v-2) t^{v-3}$. According to the assumptions $t>0$ and $0 \leq v \leq 1$, so $f_{v}^{\prime \prime}(t) \leq 0$.

The function $g_{v}(t)$ is also twice differentiable and $g_{v}^{\prime \prime}(t)=v(1-v)(v+1)\left(\frac{v t-v-2}{t^{v+3}}\right)$ which implies (ii). 
Using this lemma, together with (2.1), we have the following proposition.

Proposition 2.1. Let $A, B$ be strictly positive operators such that $A \leq B$. Then for each $0 \leq v \leq 1$

$$
\begin{aligned}
& v(B-A) A^{-1}\left(\frac{A-A \bigsqcup_{v-1} B}{2}\right)+A \sharp_{v} B \\
& \leq A \nabla_{v} B \\
& \leq v(B-A) A^{-1}\left(A-A^{\frac{1}{2}}\left(\frac{I+A^{-\frac{1}{2}} B A^{-\frac{1}{2}}}{2}\right)^{v-1} A^{\frac{1}{2}}\right)+A \sharp_{v} B .
\end{aligned}
$$

Proof. In order to prove (2.2), we firstly prove the corresponding scalar inequalities. As we showed in Lemma 2.1(i), the function $f_{v}(t)=v\left(1-t^{v-1}\right)$ where $t \geq 1$ and $0 \leq v \leq 1$ is concave. Moreover, we readily check that

$$
\int_{1}^{x} f_{v}(t) d t=(1-v)+v x-x^{v}
$$

From the inequality (2.1) for concave function we infer that

$$
v(x-1)\left(\frac{1-x^{v-1}}{2}\right)+x^{v} \leq(1-v)+v x \leq v(x-1)\left(1-\left(\frac{1+x}{2}\right)^{v-1}\right)+x^{v},
$$

where $x \geq 1$ and $0 \leq v \leq 1$.

With $X=A^{-\frac{1}{2}} B A^{-\frac{1}{2}}$ and thus $S p(X) \subseteq(1,+\infty)$, relation $(2.3)$ holds for any $x \in S p(X)$. Therefore

$$
\begin{aligned}
v(X-I)\left(\frac{I-X^{v-1}}{2}\right)+X^{v} & \leq(1-v) I+v X \\
& \leq v(X-I)\left(I-\left(\frac{I+X}{2}\right)^{v-1}\right)+X^{v} .
\end{aligned}
$$

Finally, multiplying both sides by $A^{\frac{1}{2}}$, we get (2.2).

By virtue of Proposition 2.1, we can improve the first inequality in (1.1).

Remark 2.1. It is worth remarking that the left-hand side of inequality (2.2), is a refinement of operator Young inequality in the sense of $v(x-1)\left(\frac{1-x^{v-1}}{2}\right) \geq 0$ for each $x \geq 1$ and $0 \leq v \leq 1$, 
i.e.,

$$
\begin{aligned}
& A \sharp_{v} B \\
& \leq v(B-A) A^{-1}\left(\frac{A-A \bigsqcup_{v-1} B}{2}\right)+A \sharp_{v} B \\
& \leq A \nabla_{v} B .
\end{aligned}
$$

Replacing $A$ and $B$ by $A^{-1}$ and $B^{-1}$ respectively in (2.4), we obtain

$$
\begin{aligned}
& A^{-1} \sharp_{v} B^{-1} \\
& \leq v\left(B^{-1}-A^{-1}\right) A\left(\frac{A^{-1}-A^{-1} \natural_{v-1} B^{-1}}{2}\right)+A^{-1} \sharp_{v} B^{-1} \\
& \leq A^{-1} \nabla_{v} B^{-1} .
\end{aligned}
$$

Taking inverse in (2.5), we get

$$
\begin{aligned}
& A !_{v} B \\
& \leq\left\{v\left(B^{-1}-A^{-1}\right) A\left(\frac{A^{-1}-A^{-1} \natural_{v-1} B^{-1}}{2}\right)+A^{-1} \sharp_{v} B^{-1}\right\}^{-1} \\
& \leq A \sharp_{v} B .
\end{aligned}
$$

In order to give a proof of our first main result, we need the following essential result.

Proposition 2.2. For each $0<x \leq 1$ and $0 \leq v \leq 1$, the functions $m_{v}(x)$ and $M_{v}(x)$ defined in Theorem $A$ are decreasing. Moreover $1 \leq m_{v}(x) \leq M_{v}(x)$.

Proof. The function $m_{v}(x)$ is differentiable and

$$
m_{v}^{\prime}(x)=\frac{v(v-1) 2^{v}}{(x+1)^{v+2}}\left((v-1) x^{2}+v+3-2(v+1) x\right) .
$$

By assumptions we can find easily that $m_{v}{ }^{\prime}(x) \leq 0$, for any $0<x \leq 1$ and $0 \leq v \leq 1$. In addition $m_{v}(1)=1$, so $m_{v}(x) \geq 1$.

Similarly the function $M_{v}(x)$ is differentiable and

$$
M_{v}^{\prime}(x)=\frac{v(v-1)(x-1)((v-1) x-v-1)}{2 x^{v+2}} .
$$

Therefore $M_{v}^{\prime}(x) \leq 0$ for any $0<x \leq 1$ and $0 \leq v \leq 1$. We also have $M_{v}(1)=1$, i.e., $M_{v}(x) \geq 1$. It remains to prove $m_{v}(x) \leq M_{v}(x)$. Suppose that

$$
\mathfrak{M}_{v}(x) \equiv M_{v}(x)-m_{v}(x) \quad 0<x \leq 1 \text { and } 0 \leq v \leq 1 .
$$

In a way similar to what we have done above, we can calculate $\mathfrak{M}_{v}^{\prime}(x)$ in the following:

$$
\mathfrak{M}_{v}^{\prime}(x)=\frac{v(1-v)(1-x)}{2(x+1)^{2} x^{v+2}} \mathfrak{h}_{v}(x),
$$


where

$$
\mathfrak{h}_{v}(x) \equiv 2 x^{2}\{(1-v) x+v+3\}\left(\frac{2 x}{x+1}\right)^{v}-\left\{(1-v) x^{3}+(3-v) x^{2}+(v+3) x+(v+1)\right\} .
$$

Since $0<x \leq 1,\left(\frac{2 x}{x+1}\right)^{v} \leq 1$. Thus $\mathfrak{M}_{v}^{\prime}(x)$ is bounded from the above:

$$
\mathfrak{M}_{v}^{\prime}(x) \leq \frac{v(1-v)(1-x)}{2(x+1)^{2} x^{v+2}} \mathfrak{k}_{v}(x),
$$

where

$$
\mathfrak{k}_{v}(x) \equiv(1-v) x^{3}+3(v+1) x^{2}-(v+3) x-(v+1) .
$$

By elementary calculations, we find that

$$
\mathfrak{k}_{v}^{\prime \prime}(x)=6(1-v) x+6(v+1) \geq 0, \quad \mathfrak{k}_{v}(0)=-(v+1)<0, \quad \mathfrak{k}_{v}(1)=0 .
$$

Thus we have $\mathfrak{k}_{v}(x) \leq 0$ which implies $\mathfrak{M}_{v}^{\prime}(x) \leq 0$ so that $\mathfrak{M}_{v}(x) \geq \mathfrak{M}_{v}(1)=0$. Therefore, the proposition follows.

We are now in a position to prove Theorem A which is a multiplicative type refinement and reverse for the operator Young inequality.

Proof of Theorem A. It is routine to check that the function $f_{v}(t)=\frac{v(1-v)(t-1)}{t^{v+1}}$ where $0<t \leq 1$ and $0 \leq v \leq 1$, is concave. We can verify that

$$
\int_{x}^{1} f_{v}(t) d t=1-\frac{(1-v)+v x}{x^{v}} .
$$

Hence from the inequality (2.1) we can write

$$
m_{v}(x) x^{v} \leq(1-v)+v x \leq M_{v}(x) x^{v},
$$

for each $0<x \leq 1$ and $0 \leq v \leq 1$.

Now, we shall use the same procedure as in [11, Theorem 2]. The inequality (2.6) implies that

$$
\min _{h^{\prime} \leq x \leq h \leq 1} m_{v}(x) x^{v} \leq(1-v)+v x \leq \max _{h^{\prime} \leq x \leq h \leq 1} M_{v}(x) x^{v} .
$$

Based on this inequality, one can easily see for which $X$

$$
\min _{h^{\prime} \leq x \leq h \leq 1} m_{v}(x) X^{v} \leq(1-v) I+v X \leq \max _{h^{\prime} \leq x \leq h \leq 1} M_{v}(x) X^{v} .
$$

By substituting $A^{-\frac{1}{2}} B A^{-\frac{1}{2}}$ for $X$ and taking into account that $m_{v}(x)$ and $M_{v}(x)$ are decreasing, the relation (2.7) implies

$$
m_{v}(h)\left(A^{-\frac{1}{2}} B A^{-\frac{1}{2}}\right)^{v} \leq(1-v) I+v A^{-\frac{1}{2}} B A^{-\frac{1}{2}} \leq M_{v}\left(h^{\prime}\right)\left(A^{-\frac{1}{2}} B A^{-\frac{1}{2}}\right)^{v} .
$$

Multiplying $A^{\frac{1}{2}}$ from the both sides to the inequality (2.8), we have the inequality (1.5). 
Remark 2.2. Notice that, the condition $0<h^{\prime} I \leq A^{-\frac{1}{2}} B A^{-\frac{1}{2}} \leq h I \leq I$ in Theorem $A$, can be replaced by $0<\alpha^{\prime} I \leq B \leq \alpha I \leq \beta I \leq A \leq \beta^{\prime} I$. In this case we have

$$
m_{v}(h) A \sharp_{v} B \leq A \nabla_{v} B \leq M_{v}\left(h^{\prime}\right) A \sharp_{v} B,
$$

where $h=\frac{\alpha}{\beta}$ and $h^{\prime}=\frac{\alpha^{\prime}}{\beta^{\prime}}$.

It is well-known that for each strictly positive operators $A$ and $B$ (see e.g., [13, Proposition 3.3.11]),

$$
H_{v}(A, B) \leq A \nabla B \quad 0 \leq v \leq 1 .
$$

A counterpart to the inequality (2.9) is as follows:

Remark 2.3. Assume the conditions of Theorem A. Then

$$
A \nabla B \leq \sqrt{M_{v}\left(h^{\prime 2}\right)} H_{v}(A, B) .
$$

Theorem A can be used to infer the following remark:

Remark 2.4. Assume the conditions of Theorem A. Then

$$
m_{v}(h) A !_{v} B \leq A \sharp_{v} B \leq M_{v}\left(h^{\prime}\right) A !_{v} B .
$$

The left-hand side of inequality (1.5) can be squared by a similar method as in [16, 17].

Corollary 2.1. Let $0<\alpha^{\prime} I \leq B \leq \alpha I \leq \beta I \leq A \leq \beta^{\prime} I$. Then for every normalized positive linear map $\Phi$,

$$
\Phi^{2}\left(A \nabla_{v} B\right) \leq\left(\frac{K\left(h^{\prime}\right)}{m_{v}(h)}\right)^{2} \Phi^{2}\left(A \sharp_{v} B\right)
$$

and

$$
\Phi^{2}\left(A \nabla_{v} B\right) \leq\left(\frac{K\left(h^{\prime}\right)}{m_{v}(h)}\right)^{2}\left(\Phi(A) \sharp_{v} \Phi(B)\right)^{2}
$$

where $h=\frac{\alpha}{\beta}$ and $h^{\prime}=\frac{\alpha^{\prime}}{\beta^{\prime}}$.

Proof. According to the assumptions

$$
\left(\alpha^{\prime}+\beta^{\prime}\right) I \geq \alpha^{\prime} \beta^{\prime} A^{-1}+A, \quad\left(\alpha^{\prime}+\beta^{\prime}\right) I \geq \alpha^{\prime} \beta^{\prime} B^{-1}+B,
$$

since $\left(t-\alpha^{\prime}\right)\left(t-\beta^{\prime}\right) \leq 0$ for $\alpha^{\prime} \leq t \leq \beta^{\prime}$. From these we can write

$$
\left(\alpha^{\prime}+\beta^{\prime}\right) I \geq \alpha^{\prime} \beta^{\prime} \Phi\left(A^{-1} \nabla_{v} B^{-1}\right)+\Phi\left(A \nabla_{v} B\right),
$$


where $\Phi$ is a normalized positive linear map. We have

$$
\begin{aligned}
& \left\|\Phi\left(A \nabla_{v} B\right) \alpha^{\prime} \beta^{\prime} m_{v}(h) \Phi^{-1}\left(A \sharp_{v} B\right)\right\| \\
& \leq \frac{1}{4}\left\|\Phi\left(A \nabla_{v} B\right)+\alpha^{\prime} \beta^{\prime} m_{v}(h) \Phi^{-1}\left(A \sharp_{v} B\right)\right\|^{2} \quad(\text { by }[2]) \\
& \leq \frac{1}{4}\left\|\Phi\left(A \nabla_{v} B\right)+\alpha^{\prime} \beta^{\prime} m_{v}(h) \Phi\left(A^{-1} \sharp_{v} B^{-1}\right)\right\|^{2} \quad \text { (by Choi's inequality [3, p. 41]) } \\
& \leq \frac{1}{4}\left\|\Phi\left(A \nabla_{v} B\right)+\alpha^{\prime} \beta^{\prime} \Phi\left(A^{-1} \nabla_{v} B^{-1}\right)\right\|^{2} \quad \text { (by Remark 2.2) } \\
& \leq \frac{1}{4}\left(\alpha^{\prime}+\beta^{\prime}\right)^{2} \quad(\text { by }(2.12)) .
\end{aligned}
$$

This is the same as saying

$$
\left\|\Phi\left(A \nabla_{v} B\right) \Phi^{-1}\left(A \sharp_{v} B\right)\right\| \leq \frac{K\left(h^{\prime}\right)}{m_{v}(h)},
$$

where $h=\frac{\alpha}{\beta}$ and $h^{\prime}=\frac{\alpha^{\prime}}{\beta^{\prime}}$. It is not hard to see that (2.13) is equivalent to (2.10). The proof of the inequality (2.11) goes likewise and we omit the details.

Remark 2.5. Obviously, the bounds in (2.10) and (2.11) are tighter than those in [17, Theorem 2.1], under the conditions $0<\alpha^{\prime} I \leq B \leq \alpha I \leq \beta I \leq A \leq \beta^{\prime} I$ with $h=\frac{\alpha}{\beta}$ and $h^{\prime}=\frac{\alpha^{\prime}}{\beta^{\prime}}$.

\section{Connection With Known Results}

In this section, we point out connections between our results given in Section 2 and some inequalities proved in other contexts. That is, we are now going to explain the advantages of our results. Let $0 \leq v \leq 1, r=\min \{v, 1-v\}, R=\max \{v, 1-v\}$ and $m_{v}(\cdot), M_{v}(\cdot)$ were defined as in Theorem A. As we will show in Appendix A, the following proposition explains the advantages of our results.

Proposition 3.1. The following statements are true.

(I-i) The lower bound of Proposition 2.1 improves the first inequality in (1.2), when $\frac{3}{4} \leq v \leq 1$ with $0<A \leq B$.

(I-ii) The upper bound of Proposition 2.1 improves the second inequality in (1.2), when $\frac{2}{3} \leq$ $v \leq 1$ with $0<A \leq B$.

(I-iii) The upper bound of Proposition 2.1 improves the second inequality in (1.2), when $0 \leq$ $v \leq \frac{1}{3}$ with $0<A \leq B$.

(II) The upper bound of Theorem A improves the inequality

$$
(1-v)+v x \leq x^{v} K(x)
$$

when $x^{v} \geq \frac{1}{2}$. 
(III) The upper bound of Theorem A improves the inequality given by Dragomir in [4, Theorem 1],

$$
(1-v)+v x \leq \exp (4 v(1-v)(K(x)-1)) x^{v}, \quad x>0
$$

when $0 \leq v \leq \frac{1}{2}$ and $0<x \leq 1$.

(IV) There is no ordering between Theorem $A$ and the inequalities (1.3) and (1.4).

Therefore we conclude that Proposition 2.1 and Theorem A are not trivial results. The proofs in the above mentioned are given in Appendix A.

\section{Inequalities Related to Heron Mean}

This section aims to prove new inequalities containing (1.6). These inequalities were given in Theorem B. Our main idea and technical tool are closely related to the inequalities (2.1).

Proof of Theorem B. Consider the function $f_{r, v}(t) \equiv r v t^{v-1}+(1-r) v$ where $t>0, r \in \mathbb{R}$ and $0 \leq v \leq 1$. Since the function $f_{r, v}(t)$ is twice differentiable, one can easily see that

$$
\frac{d f_{r, v}(t)}{d t}=r(v-1) v t^{v-2}, \quad \frac{d^{2} f_{r, v}(t)}{d t^{2}}=r(v-2)(v-1) v t^{v-3} .
$$

It is not hard to check that

$$
\left\{\begin{array}{ll}
\frac{d^{2} f_{r, v}(t)}{d t^{2}} \geq 0 & \text { for } r \geq 0 \\
\frac{d^{2} f_{r, v}(t)}{d t^{2}} \leq 0 & \text { for } r \leq 0
\end{array} .\right.
$$

Utilizing the inequality (2.1) for the function $f_{r, v}(t)$ we infer that

$$
g_{r, v}(x) \leq r x^{v}+(1-r)((1-v)+v x) \leq G_{r, v}(x),
$$

where

$$
\begin{aligned}
& g_{r, v}(x) \equiv v(x-1)\left\{r\left(\frac{1+x}{2}\right)^{v-1}+(1-r)\right\}+1, \\
& G_{r, v}(x) \equiv \frac{v(x-1)}{2}\left(r x^{v-1}+2-r\right)+1,
\end{aligned}
$$

for each $x \geq 1, r \geq 0$ and $0 \leq v \leq 1$. Similarly for each $0<x \leq 1, r \geq 0$ and $0 \leq v \leq 1$, we get

$$
G_{r, v}(x) \leq r x^{v}+(1-r)((1-v)+v x) \leq g_{r, v}(x) .
$$

If $x \geq 1$ and $r \leq 0$, we get

$$
G_{r, v}(x) \leq r x^{v}+(1-r)((1-v)+v t) \leq g_{r, v}(x),
$$

for each $0 \leq v \leq 1$. For the case $0<x \leq 1$ and $r \leq 0$ we have

$$
g_{r, v}(t) \leq r x^{v}+(1-r)((1-v)+v t) \leq G_{r, v}(x),
$$


for each $0 \leq v \leq 1$

Note that we equivalently obtain the operator inequalities from the scalar inequalities given in Theorem B. We here omit such expressions for simplicity.

Closing this section, we prove the ordering $\left\{(1-v)+v t^{-1}\right\}^{-1} \leq g_{r, v}(t)$ and $\left\{(1-v)+v t^{-1}\right\}^{-1} \leq$ $G_{r, v}(t)$ under some assumptions, for the purpose to show the advantages of our lower bounds given in Theorem B. It is known that

$$
\left\{(1-v)+v t^{-1}\right\}^{-1} \leq t^{v} \quad 0 \leq v \leq 1 \text { and } t>0,
$$

so that we also have interests in the ordering $g_{r, v}(t)$ and $G_{r, v}(t)$ with $t^{v}$. That is, we can show the following four propositions. The proofs are given in Appendix B.

Proposition 4.1. For $t \geq 1$ and $0 \leq v, r \leq 1$, we have

$$
\left\{(1-v)+v t^{-1}\right\}^{-1} \leq g_{r, v}(t)
$$

Proposition 4.2. For $0<t \leq 1$ and $0 \leq v, r \leq 1$, we have

$$
\left\{(1-v)+v t^{-1}\right\}^{-1} \leq t^{v} \leq g_{r, v}(t)
$$

Proposition 4.3. For $0 \leq r, v \leq 1$ and $c \leq t \leq 1$ with $c \equiv \frac{2^{7}-1}{5^{4}}$, we have

$$
\left\{(1-v)+v t^{-1}\right\}^{-1} \leq G_{r, v}(t) .
$$

Proposition 4.4. For $0 \leq v \leq 1, r \leq 1$ and $t \geq 1$, we have

$$
\left\{(1-v)+v t^{-1}\right\}^{-1} \leq t^{v} \leq G_{r, v}(t)
$$

Remark 4.1. Propositions 4.1-4.4 show that lower bounds given in Theorem $B$ are tighter than the known bound (Harmonic mean), for the cases given in Propositions 4.1-4.4. If $r=1$ in Proposition 4.1, then $g_{r, v}(t) \leq t^{v}$, for $t \geq 1$ and $0 \leq v \leq 1$. If $r=1$ in Proposition 4.3, then $G_{r, v}(t) \leq t^{v}$, for $c \leq t \leq 1$ and $0 \leq v \leq 1$. We thus find that Proposition 4.1 and Proposition 4.3 make sense for the purpose of finding the functions between $\left\{(1-v)+v t^{-1}\right\}^{-1}$ and $t^{v}$.

Remark 4.2. In the process of the proof in Proposition 4.3 we find the inequality:

$$
\frac{t^{v}+t}{2} \leq\left\{(1-v)+v t^{-1}\right\}^{-1}
$$

for $0 \leq v \leq 1$ and $c \leq t \leq 1$. Then we have the following inequalities:

$$
\frac{A \sharp_{v} B+B}{2} \leq A !_{v} B \leq A \sharp_{v} B,
$$

for $0<c A \leq B \leq A$ with $c=\frac{2^{7}-1}{5^{4}}$, and $0 \leq v \leq 1$. 
In the process of the proof in Proposition 4.2 we also find the inequality:

$$
t\left(\frac{t+1}{2}\right)^{v-1} \leq\left\{(1-v)+v t^{-1}\right\}^{-1}
$$

for $0 \leq v \leq 1$ and $0 \leq t \leq 1$. Then we have the following inequalities:

$$
B A^{-1 / 2}\left(\frac{A^{-1 / 2} B A^{-1 / 2}+I}{2}\right)^{v-1} A^{1 / 2} \leq A !_{v} B \leq A \sharp_{v} B,
$$

for $0<B \leq A$ and $0 \leq v \leq 1$

\section{Concluding Remark}

Several refinements and generalizations of the inequality (2.1) have been given (see, e.g. $[5,6,19,22])$. Of course, if we apply them with similar considerations were discussed above, we can find new results concerning mean inequalities. We leave the details of this idea to the interested reader, as it is just an application of our main results.

Acknowledgment. The authors thank anonymous referees for giving valuable comments and suggestions to improve our manuscript. The author (S.F.) was partially supported by JSPS KAKENHI Grant Number 16K05257

\section{REFERENCES}

[1] R. Bhatia, Interpolating the arithmetic-geometric mean inequality and its operator version, Linear Algebra Appl., 413(2-3) (2006), 355-363.

[2] R. Bhatia, F. Kittaneh, Notes on matrix arithmetic-geometric mean inequalities, Linear Algebra Appl., 308 (2000), 203-211.

[3] R. Bhatia, Positive definite matrices, Princeton University Press, Princeton, 2007.

[4] S.S. Dragomir, A note on Young's inequality, Rev. R. Acad. Cienc. Exactas Fís. Nat. Ser. A Math., 111(2) (2017), 349-354.

[5] A. El Farissi, Simple proof and refinement of Hermite-Hadamard inequality, J. Math. Inequal., 4(3) (2010), $365-369$.

[6] Y. Feng, Refinements of the Heinz inequalities, J. Inequal. Appl., (2012), Art. no. 18 (6 pp.).

[7] M. Fujii, S. Furuichi, R. Nakamoto, Estimations of Heron means for positive operators, J. Math. Inequal., 10(1) (2016), 19-30.

[8] S. Furuichi, N. Minculete, Alternative reverse inequalities for Young's inequality, J. Math Inequal., 5(4) (2011), 595-600.

[9] S. Furuichi, On refined Young inequalities and reverse inequalities, J. Math. Inequal., 5(1) (2011), 21-31.

[10] S. Furuichi, Operator inequalities among arithmetic mean, geometric mean and harmonic mean, J. Math. Inequal., 8(3) (2014), 669-672.

[11] S. Furuichi, Refined Young inequalities with Specht's ratio, J. Egyptian Math. Soc., 20(1) (2012), 46-49. 
[12] T. Furuta, M. Yanagida, Generalized means and convexity of inversion for positive operators, Amer. Math. Monthly., 105(3) (1998), 258-259.

[13] F. Hiai, Matrix Analysis: Matrix monotone functions, matrix means, and majorization, Interdisciplinary Information Science, 46(2) (2010), 139-248.

[14] F. Kittaneh, Y. Manasrah, Reverse Young and Heinz inequalities for matrices, Linear Multilinear Algebra., 59 (2011), 1031-1037.

[15] W. Liao, J. Wu, J. Zhao, New versions of reverse Young and Heinz mean inequalities with the Kantorovich constant, Taiwanese J. Math., 19(2) (2015), 467-479.

[16] M. Lin, On an operator Kantorovich inequality for positive linear maps, J. Math. Anal. Appl., 402 (2013), 127-132.

[17] M. Lin, Squaring a reverse AM-GM inequality, Studia Math., 215 (2013), 189-194.

[18] H.R. Moradi, S. Furuichi, N. Minculete, Estimates for Tsallis relative operator entropy, Math. Ineq. Appl., 20(4) (2017), 1079-1088.

[19] C.P. Niculescu, L.E. Persson, Old and new on the Hermite-Hadamard inequality, Real Anal. Exchange., 29(2) (2004), 663-686.

[20] M. Sababheh, D. Choi, A complete refinement of Young's inequality, J. Math. Anal. Appl., 440(1) (2016), 379-393.

[21] M. Sababheh, M.S. Moslehian, Advanced refinements of Young and Heinz inequalities, J. Number Theory., 172, 178-199.

[22] L. Wang, On extensions and refinements of Hermite-Hadamard inequalities for convex functions, Math. Inequal. Appl., 6(4) (2003), 659-666.

[23] K. Yanagi, K. Kuriyama, S. Furuichi, Generalized Shannon inequalities based on Tsallis relative operator entropy, Linear Algebra Appl., 394 (2005), 109-118.

[24] H. Zuo, G. Shi, M. Fujii, Refined Young inequality with Kantorovich constant, J. Math. Inequal., 5(4) (2011), 551-556.

\section{Appendix A}

For the purpose to give proof of Proposition 3.1, we need the following lemma.

Lemma 4.1. For each $x \geq 1$, we have

$$
\left(\frac{x+1}{2}\right)^{2 / 3} \geq\left(\frac{\sqrt{x}+1}{2 \sqrt{x}}\right)\left(1+\log \left(\frac{x+1}{2}\right)\right) .
$$

Proof. We firstly prove

$$
\left(\frac{x+1}{2}\right)^{2 / 3} \geq\left(\frac{1}{2}+\frac{x+1}{4 x}\right)\left(1+\log \left(\frac{x+1}{2}\right)\right),
$$

for $x \geq 1$. Putting $t=\frac{x+1}{2} \geq 1$, the inequality (4.12) is equivalent to the inequality

$$
t^{2 / 3} \geq \frac{(3 t-1)}{2(2 t-1)}(1+\log t)
$$


which is equivalent to saying

$$
2 s^{2}\left(2 s^{3}-1\right) \geq\left(3 s^{3}-1\right)(1+3 \log s),
$$

where $s=t^{1 / 3} \geq 1$. To prove the above inequality, we set

$$
\mathfrak{F}(s) \equiv 4 s^{5}-3 s^{3}-2 s^{2}+1-9 s^{3} \log s+3 \log s \quad s \geq 1 .
$$

By simple calculations, we have $\mathfrak{F}(s) \geq \mathfrak{F}(1)=0$. Hence we have the inequality (4.12). For any $a>0$, we have $\frac{2 a}{1+a} \leq \sqrt{a}$, that is, $\frac{a+1}{2 a} \geq \frac{1}{\sqrt{a}}$. Therefore for any $a>0$, we have $\frac{1}{2}+\frac{a+1}{4 a} \geq \frac{1}{2}+\frac{1}{2 \sqrt{a}}=\frac{\sqrt{a}+1}{2 \sqrt{a}}$, which implies the following second inequality

$$
\left(\frac{x+1}{2}\right)^{2 / 3} \geq\left(\frac{1}{2}+\frac{x+1}{4 x}\right)\left(1+\log \left(\frac{x+1}{2}\right)\right) \geq\left(\frac{\sqrt{x}+1}{2 \sqrt{x}}\right)\left(1+\log \left(\frac{x+1}{2}\right)\right) .
$$

This completes the proof.

Proof of Proposition 3.1.

(I) Assume that $x \geq 1$.

(i) Consider the function

$$
u_{v}(x) \equiv v(x-1)\left(\frac{1-x^{v-1}}{2}\right)-r(1-\sqrt{x})^{2} .
$$

For $\frac{3}{4} \leq v \leq 1$, we have $u_{v}(x) \geq 0$. Let us prove this statement. Since $u_{1}(x)=0$ and $\frac{d^{2} u_{v}(x)}{d v^{2}}=\frac{1}{2}(1-x) x^{v-1}\left\{2 \log x+v(\log x)^{2}\right\} \leq 0$ for $x \geq 1$, we have only to prove $u_{3 / 4}(x) \geq 0$ for $x \geq 1$. Since $u_{3 / 4}(x)=\frac{x^{5 / 4}-3 x+4 x^{3 / 4}-5 x^{1 / 4}+3}{8 x^{1 / 4}}$, we set the function $\mathfrak{v}(x) \equiv x^{5 / 4}-3 x+4 x^{3 / 4}-5 x^{1 / 4}+3$. Some calculations show $\mathfrak{v}(x) \geq \mathfrak{v}(x)=0$ which implies $u_{3 / 4}(x) \geq 0$. Hence our claim follows.

In this case, the first inequality in (2.2), can be considered as a refinement of the first inequality in (1.2).

(ii) Consider the function

$$
w_{v}(x) \equiv R(1-\sqrt{x})^{2}-v(x-1)\left(1-\left(\frac{x+1}{2}\right)^{v-1}\right) .
$$

For $\frac{2}{3} \leq v \leq 1$, we have $w_{v}(x) \geq 0$. For proving this inequality, let $\mathfrak{x}_{v}(x)=$ $(1-\sqrt{x})^{2}-(x-1)\left(1-\left(\frac{x+1}{2}\right)^{v-1}\right)$. For $x \geq 1$, we then have $\frac{d \mathfrak{x}_{v}(x)}{d v}=(x-$ 1) $\left(\frac{x+1}{2}\right)^{v-1}\left\{\log \left(\frac{x+1}{2}\right)\right\} \geq 0$. We have only to prove $\mathfrak{x}_{2 / 3}(x) \geq 0$ for $x \geq 1$. By slightly complicated calculations, we have

$$
\mathfrak{x}_{2 / 3}(x)=\frac{2^{4 / 3}(\sqrt{x}-1)}{(x+1)^{1 / 3}}\left\{\frac{\sqrt{x}+1}{2}-\left(\frac{x+1}{2}\right)^{1 / 3}\right\} \geq 0 .
$$


Indeed, for $t \geq 1$, we have $(t-1)\left(t^{2}+3\right) \geq 0$ which is equivalent to $(t+1)^{3} \geq 4\left(t^{2}+1\right)$. Putting $t=\sqrt{x}$, we obtain $\frac{(\sqrt{x}+1)^{3}}{8} \geq \frac{x+1}{2}$ which shows $\frac{\sqrt{x}+1}{2} \geq\left(\frac{x+1}{2}\right)^{1 / 3}$. Thus our assertion follows.

(iii) In addition, for $0 \leq v \leq \frac{1}{3}$, we have $w_{v}(x) \geq 0$. In fact, since $v\left(\frac{x+1}{2}\right)^{v-1}$ is increasing for $v$, we estimate the first derivative of $w_{v}(x)$ as

$$
\begin{aligned}
& \frac{d w_{v}(x)}{d v}=-(\sqrt{x}-1)^{2}-(x-1)+(x-1)\left(\frac{x+1}{2}\right)^{v-1}\left(1+v \log \left(\frac{x+1}{2}\right)\right) \\
& \leq-(\sqrt{x}-1)^{2}-(x-1)+(x-1)\left(\frac{x+1}{2}\right)^{-2 / 3}\left(1+\frac{1}{3} \log \left(\frac{x+1}{2}\right)\right) \\
& =-\frac{2^{5 / 3} \sqrt{x}(\sqrt{x}-1)}{(x+1)^{2 / 3}}\left\{\left(\frac{x+1}{2}\right)^{2 / 3}-\frac{\sqrt{x}+1}{2 \sqrt{x}}\left(1+\log \left(\frac{x+1}{2}\right)\right)\right\}
\end{aligned}
$$

$\leq 0$.

The last inequality is due to Lemma 4.1. Consequently, $w_{v}(x) \geq w_{1 / 3}(x)$. So we prove $w_{1 / 3}(x) \geq 0$. After short computations, we get

$$
w_{1 / 3}(x)=\frac{\sqrt{x}-1}{3}\left(\frac{x+1}{2}\right)^{-2 / 3}\left\{(\sqrt{x}-3)\left(\frac{x+1}{2}\right)^{2 / 3}+\sqrt{x}+1\right\} .
$$

Now we set the function $\mathfrak{y}(t) \equiv(t-3)\left(\frac{t^{2}+1}{2}\right)^{2 / 3}+t+1$ for $t \geq 1$. By some calculations, we get $\mathfrak{y}(t) \geq \mathfrak{y}(1)=0$. Therefore we have $w_{v}(t) \geq w_{1 / 3}(t) \geq 0$, as required.

In this cases, the second inequality in (2.2) provides an improvement for the second inequality in $(1.2)^{1}$.

(II) Let $x>0$. It is clear that if $x^{v} \geq \frac{1}{2}$, then $M_{v}(x) \leq K(x)$. Indeed, by simple calculations, the inequality $M_{v}(x) \leq K(x)$ is equivalent to the inequality $2 v(1-v) \leq x^{v}$. Since $v(1-v) \leq \frac{1}{4}$, we have $x^{v} \geq \frac{1}{2} \geq 2 v(1-v)$ under the condition $x^{v} \geq \frac{1}{2}$.

(III) Dragomir obtained the inequality (3.1) in [4, Theorem 1] for $x>0$. However, for $0 \leq v \leq \frac{1}{2}$ and $0<x \leq 1$, we show

$$
M_{v}(x) \leq \exp (4 v(1-v)(K(x)-1))
$$

Our upper bound of Theorem $\mathrm{A}$ is tighter than one given in [4, Theorem 1], when $0 \leq v \leq \frac{1}{2}$.

\footnotetext{
${ }^{1}$ It is interesting to note that, by the computer calculations, we find that if $v \geq 0.7$ then $u_{v}(x) \geq 0$ and if $v \geq 0.6$ or $v \leq 0.4$ we have $w_{v}(x) \geq 0$. These mean we have a possibility to extend the range of $v$ to satisfy the condition of (I-i), (I-ii) and (I-iii) in Proposition 3.1.
} 
Let us prove the above inequality (4.13) which is equivalent to the inequality

$$
1+\frac{1}{2 x^{v}} \frac{v(1-v)(x-1)^{2}}{x} \leq \exp \left(\frac{v(1-v)(x-1)^{2}}{x}\right) .
$$

We use the inequality

$$
\exp (y) \geq 1+y+\frac{1}{2} y^{2}, \quad y \geq 0
$$

with $y=\frac{v(1-v)(x-1)^{2}}{x} \geq 0$. Then we calculate

$$
\begin{aligned}
& \exp \left(\frac{v(1-v)(x-1)^{2}}{x}\right)-1-\frac{1}{2 x^{v}} \frac{v(1-v)(x-1)^{2}}{x} \\
& \geq \frac{v(1-v)(x-1)^{2}}{x}\left(1-\frac{1}{2 x^{v}}+\frac{v(1-v)(x-1)^{2}}{2 x}\right) \\
& =\frac{v(1-v)(x-1)^{2}}{x}\left(\frac{2 x^{v}-1+v(1-v) x^{v-1}(x-1)^{2}}{2 x^{v}}\right) .
\end{aligned}
$$

Thus we have only to prove $2 x^{v}-1+v(1-v) x^{v-1}(x-1)^{2} \geq 0$ for $0<x \leq 1$ and $0 \leq v \leq \frac{1}{2}$. By putting $t=1 / x$, the above inequality becomes

$$
t^{-v-1}\left(2 t-t^{v+1}+v(1-v)(t-1)^{2}\right) \geq 0 .
$$

Therefore it is sufficient to prove the inequality

$$
\mathfrak{g}_{v}(t) \equiv 2 t-t^{v+1}+v(1-v)(t-1)^{2} \geq 0,
$$

for $t \geq 1$ and $0 \leq v \leq \frac{1}{2}$. By some calculations, we have $\mathfrak{g}_{v}(t) \geq \mathfrak{g}_{1 / 2}(t) \geq \mathfrak{g}_{1 / 2}(1)=1>$ 0 . Thus the proof of the inequality (4.13) was completed.

It should be mentioned here that the inequality (4.13) holds for $0 \leq v \leq 1$ and $x \geq \frac{1}{2}$ from (4.14).

(IV) It is natural to consider $m_{v}(x)$ and $M_{v}(x)$ are better than $K^{r}(x)$ and $K^{R}(x)$ under the assumption $0<x \leq 1$.

(i) In general, there is no ordering between $K^{r}(x)$ and $m_{v}(x)$. For this purpose, taking $v=0.3$ and $x=0.7$, then

$$
m_{v}(x)-K^{r}(x) \approx 0.002 .
$$

On the other hand, taking $v=0.7$ and $x=0.1$, we have

$$
m_{v}(x)-K^{r}(x) \approx-0.15 \text {. }
$$

(ii) In addition, we have no ordering between $K^{R}(x)$ and $M_{v}(x)$. To see this putting $v=0.2$ and $x=0.4$, observe that

$$
K^{R}(x)-M_{v}(x) \approx 0.08 .
$$


But if we choose $v=0.6$ and $x=0.3$ we get

$$
K^{R}(x)-M_{v}(x) \approx-0.17
$$

\section{Appendix B}

Proof of Proposition 4.1. Since $g_{r, v}(t)$ is decreasing in $r, g_{r, v}(t) \geq g_{1, v}(t)$ so that we have only to prove for $t \geq 1$ and $0 \leq v \leq 1$, the inequality $g_{1, v}(t) \geq\left\{(1-v)+v t^{-1}\right\}^{-1}$ which is equivalent to the inequality by $v(t-1) \geq 0$

$$
\left(\frac{t+1}{2}\right)^{v-1} \geq \frac{1}{(1-v) t+v}
$$

Since $t \geq 1$ and $0 \leq v \leq 1$, we have $t\left(\frac{t+1}{2}\right)^{v-1} \geq t^{v}$. In addition, for $t>0$ and $0 \leq v \leq 1$, we have $t^{v} \geq\left\{(1-v)+v t^{-1}\right\}^{-1}$. Thus we have $t\left(\frac{t+1}{2}\right)^{v-1} \geq\left\{(1-v)+v t^{-1}\right\}^{-1}$ which implies the inequality (4.15).

Proof of Proposition 4.2. The first inequality is know for $t>0$ and $0 \leq v \leq 1$. Since $g_{r, v}(t)$ is deceasing in $r$, in order to prove the second inequality we have only to prove $g_{1, v}(t) \geq t^{v}$, that is,

$$
v(t-1)\left(\frac{t+1}{2}\right)^{v-1}+1 \geq t^{v} .
$$

which is equivalent to the inequality

$$
\frac{t^{v}-1}{v} \leq(t-1)\left(\frac{t+1}{2}\right)^{v-1} .
$$

By the use of Hermite-Hadamard inequality with a convex function $x^{v-1}$ for $0 \leq v \leq 1$ and $x>0$, the above inequality can be proven as

$$
\left(\frac{t+1}{2}\right)^{v-1} \leq \frac{1}{1-t} \int_{t}^{1} x^{v-1} d x=\frac{1-t^{v}}{v(1-t)}
$$

Proof of Proposition 4.3. We firstly prove $\mathrm{h}(t) \equiv 2(t-1)-\log t \geq 0$ for $c \leq t \leq 1$. Since $\mathrm{h}^{\prime \prime}(t) \geq 0, \mathrm{~h}(1)=0$ and $\mathrm{h}(c) \approx-0.0000354367<0$. Thus we have $\mathrm{h}(t) \leq 0$ for $c \leq t \leq 1$. Secondly we prove $\mathrm{I}_{v}(t) \equiv 2(t-1)-((1-v) t+v) \log t \leq 0$. Since $\frac{d \mathrm{l}_{v}(t)}{d v}=(t-1) \log t \geq 0$, we have $\mathrm{I}_{v}(t) \leq \mathrm{I}_{1}(t)=\mathrm{h}(t) \leq 0$. Since $G_{r, v}(t)$ is decreasing in $r$, we have $G_{r, v}(t) \geq G_{1, v}(t)$ so that we have only to prove $G_{1, v}(t) \geq\left\{(1-v)+v t^{-1}\right\}^{-1}$, which is equivalent to the inequality, by $v(t-1) \leq 0$

$$
\frac{t^{v-1}+1}{2} \leq \frac{1}{(1-v) t+v}
$$


for $0 \leq r, v \leq 1$ and $c \leq t \leq 1$. To this end, we set $\mathrm{f}_{v}(t) \equiv 2-\left(t^{v-1}+1\right)((1-v) t+v)$. Some calculations imply $\mathrm{f}_{v}(t) \geq \mathrm{f}_{1}(t)=0$.

Proof of Proposition 4.4. The first inequality is know for $t>0$ and $0 \leq v \leq 1$. Since $G_{r, v}(t)$ is deceasing in $r$, in order to prove the second inequality we have only to prove $G_{1, v}(t) \geq t^{v}$, which is equivalent to the inequality

$$
\frac{1}{2} v(t-1)\left(t^{v-1}+1\right)+1 \geq t^{v}
$$

To this end, we set

$$
\mathbf{k}_{v}(t) \equiv v(t-1)\left(t^{v-1}+1\right)+2-2 t^{v} .
$$

Some calculations imply $\mathrm{k}_{v}(t) \geq \mathrm{k}_{v}(1)=0$.

${ }^{1}$ Department of Information Science, College of Humanities and Sciences, Nihon University, 3-25-40, Sakurajyousui, Setagaya-ku, Tokyo, 156-8550, Japan.

E-mail address: furuichi@chs.nihon-u.ac.jp

${ }^{2}$ Young Researchers and Elite Club, Mashhad Branch, Islamic Azad University, Mashhad, Iran.

E-mail address: hrmoradi@mshdiau.ac.ir 